\title{
The Lateral Femoral Wall Thickness on the Risk of Post-Operative Lateral Wall Fracture in Intertrochanteric Fracture: A Finite Element Analysis
}

\section{Jixing Fan}

Peking University Third Hospital

\section{Xiangyu Xu}

Peking University Third Hospital

Fang Zhou ( $\square$ zhouf@bjmu.edu.cn )

Peking University Third Hospital

\section{Research article}

Keywords: Intertrochanteric fracture, Lateral femoral wall thickness, Dynamic hip screw(DHS), Finite element analysis

Posted Date: July 19th, 2021

DOl: https://doi.org/10.21203/rs.3.rs-682386/v1

License: (c) (i) This work is licensed under a Creative Commons Attribution 4.0 International License. Read Full License 


\section{Abstract}

Background Patients with a lateral femoral wall (LFW) fracture were reported to have high rates of reoperation and complication. Although the LFW thickness was a reliable predictor of post-operative or intra-operative LFW fracture, no biomechanical studies had evaluated the critical stress distributions on the femur and screws of intertrochanteric fractures treated with dynamic hip screw (DHS). This study aimed to investigate the biomechanical performance of intertrochanteric fractures with different LFW thickness treated with DHS device.

Methods A three-dimensional model of the proximal femur was established by computed tomography images. The intertrochanteric fracture model with three different LFW thickness $(10 \mathrm{~mm}, 20.5 \mathrm{~mm}$ and $30 \mathrm{~mm}$ respectively) was created, which was fixed by DHS. The von Mises stress on the proximal femur, lateral femoral wall, DHS and the total displacement of the device components were evaluated and compared for three different LFW thickness model.

Results The maximum von Mises stress in the proximal fragment of the $10 \mathrm{~mm}$ and $20.5 \mathrm{~mm}$ model increased by $80.56 \%$ and $57.97 \%$ when compared with the $30 \mathrm{~mm}$ model. The peek von Mises stress around the blade entry point of the $10 \mathrm{~mm}$ and $20.5 \mathrm{~mm}$ model increased by $89.26 \%$ and $66.39 \%$ when compared with the $30 \mathrm{~mm}$ model. The peek von Mises in the DHS located near the junction of the barrel and side plate of each model and the $30 \mathrm{~mm}$ model had the smallest von Mises stress compared with the other two models. Furthermore, the maximum displacement in the $30 \mathrm{~mm}$ model was much smaller than that in the $10 \mathrm{~mm}$ model and $20 \mathrm{~mm}$ model.

Conclusions The intertrochanteric fracture with a thinner LFW tended to have a higher risk of LFW fracture stabilized by a DHS device. Thus, the intertrochanteric fractures with a thinner LFW should not be treated by DHS alone and the intramedullary nail or an addition of trochanteric stabilization plate(TSP) was recommended.

\section{Introduction}

Intertrochanteric fractures, accounting for about $40 \%$ of hip fractures, pose an important public health problem[1]. Along with the growing number of the elderly population, the incidence of these fractures continues to increase worldwide. Intertrochanteric fractures are associated with significant morbidity and mortality, which would place a heavy financial burden on the health-care system[2, 3]. A substantial part of this cost is directly related to the cost of the implants. The clinical efficacy of more costly implants should be objectively evaluated before their general use. The dynamic hip screw(DHS) has the advantage of having a lower cost. The integrity of the lateral femoral wall (LFW) on the pre-operative radiograph had been recommended to be a major factor in determining the internal fixation device used for fracture stabilization[4, 5].

The intact LFW, anatomically defined as the lateral femoral cortex distal to the vastus ridge, plays a key role in stabilization of unstable intertrochanteric fractures by providing a lateral buttress for the proximal 
fragment, and its deficiency could lead to excessive collapse and varus malpositioning[6, 4]. DHS was considered as the gold standard implant for the intertrochanteric fracture fixation for many advantages such as controlled impaction and short operation time. However, it had also been reported that significant medial displacement of the shaft resulting from excessive sliding of screw within the barrel and a higher incidence of screw cut-out after an intertrochanteric fracture treated with a sliding compression hip screw[5]. The possible reason might be that the broken LFW could result in the lack of buttress support of the lateral wall. Therefore, the identification of patients at a higher risk of a secondary lateral wall fracture would greatly improve the outcome of DHS treatment.

Previous studies had reported that the LFW thickness was a reliable predictor of post-operative or intraoperative LFW fracture[7, 8]. Furthermore, they concluded that intertrochanteric fractures with a LFW thickness less than 20.5 or $21 \mathrm{~mm}$ should not be treated with DHS alone. We hypothesized that the possible reason might be the LFW was too thin to provide sufficient lateral buttress for the proximal fragment. Furthermore, the large-diameter hole drilled into the LFW would weaken the strength of the LFW. However, no biomechanical studies had evaluated the critical stress distributions on the femur and screws. In present study, we used finite element analysis (FEA) to investigate the impact of different LFW thickness on the risk of post-operative lateral wall fracture in intertrochanteric fracture after DHS fixation. This promising research was the first to numerically investigate critical stress distributions on the femur and screws for intertrochanteric fractures with different LFW thickness; therefore, it was believed that these results would encourage future investigations into fracture mechanisms and implant selection.

\section{Methods}

\section{Finite element model establishment}

In present study, one healthy Chinese male volunteer was recruited: age 65 years old, weight $70 \mathrm{~kg}$, height $170 \mathrm{~cm}$. The X-ray examination was performed, which showed that the femur was normal without any signs of femoral diseases or deformities. The proximal femur was scanned with a 64-slice spiral CT (GE, USA), and the data were saved in Digital Imaging and Communications in Medicine (DICOM) format. Then, the femur data was imported into Mimics 17.0 software (Materialise, Belgium) and the threedimensional (3D) model of the proximal femur was reconstructed from the CT images. The surface errors (spike, intersection, etc.) of the 3D model of the proximal femur were corrected in the Geomagic Studio 12.0 software (Raindrop Inc., USA). After the correction of the surface roughness of the model, the 3D smooth solid model was developed and imported into SolidWorks program (Dassault Systemes SolidWorks Corp., USA). Next, the intertrochanteric fracture model with three different LFW thickness $(10 \mathrm{~mm}, 20.5 \mathrm{~mm}$ and $30 \mathrm{~mm}$ respectively) was created using the SolidWorks.(Fig. 1)

Then, the 3D model of DHS was established according to DePuy-Synthes DHS via the SolidWorks software. (Fig. 2B) The geometrical dimensions of the DHS with a $130^{\circ}$ four-hole standard barrel plate were obtained from the implant manufacturer catalogue. Then, the DHS was virtually inserted into the proximal femur and the tip-apex distance (TAD) was controlled within $25 \mathrm{~mm}$. Finally, the proximal femur 
models with DHS implants were imported into ANSYS Workbench 14.5 (ANSYS Inc., Canonsburg, PA) for analysis. (Fig. 3)

The solid models were discretized into four-node tetrahedral elements using ANSYS Workbench. To evaluate the accuracy of finite element models, convergence tests were performed to determine the optimum maximum element size. After the convergence measurement, the mesh size was determined to be $2 \mathrm{~mm}$.

In present study, all materials were assumed to be homogeneous, isotropic, and with linear elastic behavior[9]. The material properties of the femur and implant materials used in the models were summarized in Table $1[10,11]$. A friction contact was used for describing the contact interactions between bone fragments, between implant components, and between bone and implants. The friction coefficient was set to be 0.46 [12] between the fracture surfaces, 0.23 between implant components[13] and 0.30 between bone and implants[14].

Table 1

Material properties used in the simulations in this study

\begin{tabular}{|l|lll|}
\cline { 2 - 4 } & Material & Young's modulus (Mpa) & Poisson's ratio \\
\cline { 2 - 4 } & Cortical bone & 17000 & 0.33 \\
\hline Cancellous bone & 1000 & 0.3 \\
\hline DHS (Ti-6Al-7NB) & 110000 & 0.35 \\
\hline
\end{tabular}

For boundary condition, the distal end of the femur was constrained in all degrees of freedom. The loading forces acting on the femur presented the loads at the heel strike of normal walking[15]. A joint reaction force of $2967.7 \mathrm{~N}(\{x, y, z\}=\{1234.8,-352.8,-2675.4\})$ was applied at the femoral head( 4.2 times body weight). To reduce bending moments at the proximal femur, an abductor force was applied on the greater trochanter[16]. An abductor muscle load of $1288.3 \mathrm{~N}(\{x, y, z\}=\{-460.6,634.34,1022.28\})$ was applied at the greater trochanter ( 1.9 times body weight). (Fig. $2 \mathrm{~A})$

\section{Results}

In present study, the total displacement and the peak von Mises stress were selected as indices of the stability of a fractured femur stabilized by a DHS device and of the risk of implant failure, respectively. The von Mises stress on the proximal femur, lateral femoral wall, DHS and the total displacement of the device components were evaluated and compared for three different LFW thickness model $(10 \mathrm{~mm}$, $20.5 \mathrm{~mm}$ and $30 \mathrm{~mm}$ ) under the heel strike of normal walking.

\section{Von Mises stress in the proximal femur}


The von Mises stress distribution of the proximal femur for different LFW thickness were shown in Fig. 4. In the three models, the stress concentration area was located at the medial-inferior part of the proximal femur; while differences in von Mises stress distribution were observed. The maximum von Mises stress of the $10 \mathrm{~mm}$ model and $20.5 \mathrm{~mm}$ model increased by $80.56 \%$ and $57.97 \%$ when compared with the $30 \mathrm{~mm}$ model, respectively. The $30 \mathrm{~mm}$ model had the smallest peak von Mises stress compared with the other two models, and the magnitude was approximately $62.01 \mathrm{MPa}$. The $10 \mathrm{~mm} \mathrm{LFW}$ thickness model had the largest peek von Mises stress and the 20.5mm LFW thickness model had the slightly smaller peek von Mises stress compared with $30 \mathrm{~mm}$ model, and the magnitude for these two models were 111.96MPa and $97.95 \mathrm{MPa}$, respectively.

\section{Von Mises stress distribution around blade entry point}

Figure 5 showed the von Mises stress distribution around blade entry point for different LFW thickness. The peek von Mises stress was located at the blade entry point of the LFW in all of the three models. The results showed that the $30 \mathrm{~mm}$ model had the smallest peek van Mises stress compared with the other two models. The peek von Mises stress of the $10 \mathrm{~mm}$ model and $20.5 \mathrm{~mm}$ model increased by $89.26 \%$ and $66.39 \%$ when compared with the $30 \mathrm{~mm}$ model, respectively. The magnitude of peek von Mises stress in $10 \mathrm{~mm}, 20.5 \mathrm{~mm}$ and $30 \mathrm{~mm}$ model was $113.97 \mathrm{MPa}, 100.20 \mathrm{MPa}$ and $60.22 \mathrm{MPa}$, respectively.

\section{Von Mises stress distribution in the DHS}

The von Mises stress distribution was investigated for DHS with different LFW thickness as shown in Fig. 6. The peek von Mises in the DHS located near the junction of the barrel and side plate of each model. The $10 \mathrm{~mm}$ model and $20.5 \mathrm{~mm}$ model had similar peek von Mises stress, and the magnitude was 368.18MPa and $360.03 \mathrm{MPa}$, respectively. The $30 \mathrm{~mm}$ model had the smallest von Mises stress compared with the other two models, and the magnitude was 249.58MPa.

\section{Model displacement}

Figure 7 depicted the model displacement distribution in three models with different LFW thickness. The maximum displacement were located at the top of the femoral head for all of the three models. The $30 \mathrm{~mm}$ model showed smallest maximum displacement compared with the $10 \mathrm{~mm}$ and $20.5 \mathrm{~mm}$ model. The maximum amount of displacement of the three models ( $10 \mathrm{~mm}$ model, $20.5 \mathrm{~mm}$ model and $30 \mathrm{~mm}$ model) was $5.19 \mathrm{~mm}, 5.00 \mathrm{~mm}$ and $4.30 \mathrm{~mm}$, respectively.

\section{Discussion}

Intertrochanteric fracture of the proximal femur was a common osteoporotic fracture in the elderly[17, 18]. The optimal treatment and implant device selection for the intertrochanteric fracture remained controversial[19, 20]. DHS was a common implant used to treat stable-type intertrochanteric hip fractures. However, a few studies reported that the DHS should be avoided in the intertrochanteric fracture with a 
broken LFW for the high incidence of reoperation[5, 4]. Furthermore, Hsu.et al[7] reported that the lateral wall thickness was a reliable predictor of post-operative lateral wall fracture and concluded that intertrochanteric fractures with a lateral wall thickness $<20.5 \mathrm{~mm}$ should not be treated with DHS alone. Another study also showed that the lateral wall thickness was a reliable predictor of intraoperative lateral wall fracture during DHS fixation[8]. We thought that the thinner LFW was too weak to provide sufficient lateral buttress for the proximal fragment, and the large-diameter hole drilled into the LFW would be another factor contributing to weaken the strength of the LFW. Nevertheless, to the author's knowledge, there were no studies relating to simulation and comparison of biomechanical performance of DHS fixation with different LFW thickness.

Finite element analysis was a common tool used in biomechanical validation studies of orthopedic or dental implants. In present study, we constructed 3D finite element models with different LFW thickness of the intertrochanteric fractures treated with DHS to compare the differences in biomechanical properties. Our results showed that the stress of the proximal femur, LFW and implant in the $10 \mathrm{~mm}$ model were higher than that in $20.5 \mathrm{~mm}$ model and $30 \mathrm{~mm}$ model. Furthermore, the maximum displacement in the $30 \mathrm{~mm}$ model was much smaller than that in the $10 \mathrm{~mm}$ model and $20 \mathrm{~mm}$ model, and the maximum displacement in the $10 \mathrm{~mm}$ model and $20 \mathrm{~mm}$ model was similar.

The LFW fracture was an important predictor of a reoperation in the intertrochanteric fracture treated with a sliding compression hip-screw device. Palm et al.[5] investigated 214 consecutive patients with an intertrochanteric fracture treated with a $135^{\circ}$ sliding compression hip screw and the results showed that patients with a fractured LFW underwent a significant higher reoperation rates than those with an intact LFW postoperatively within six months $(22 \%$ versus $3 \%, \mathrm{p}<0.001)$. Moreover, $74 \%$ (thirty-four) of the forty-six fractures of the LFW occurred during the operative procedure itself. Another study revealed that $19.5 \%$ (thirty-four) of the 135 patients underwent LFW fractures during surgery[8]. Both of these two studies revealed that a compromised lateral femoral wall was a reliable predictor of intraoperative or post-operative LFW fracture in the intertrochanteric fracture. In present study, we found that the stress concentration in the LFW located at the area around the blade entry point in all of the three models. Furthermore, we discovered that the thinner of the LFW thickness, the greater the stress on the cortical bone of the lateral femoral wall. The peek von Mises stress of the $10 \mathrm{~mm}$ model and $20.5 \mathrm{~mm}$ model increased by $89.26 \%$ and $66.39 \%$ when compared with the $30 \mathrm{~mm}$ model, respectively. One reason might be that the high-quality LFW could prevent excessive sliding of the proximal fragment and reduce the stress concentration significantly when compared with the comprised LFW in the intertrochanteric fracture after DSH fixation. Therefore, we thought that the intertrochanteric fractures with a comprised LFW would have a higher risk of intraoperative or postoperative LFW fracture after DHS fixation and should not be treated by DHS alone.

The loss of integrity of the medial or lateral wall of an intertrochanteric region had been suggested as the most important cause of instability in intertrochanteric fractures $[5,21,22]$. The medial wall consisted of the medial cortex and medial calcar located in its deep side. The loss of the medial support had been proven to cause coxa varus postoperatively due to the hinge[23]. When a lateral wall fracture occurred, 
the medial support played a important role in maintaining the stability of the intertrochanteric fracture. Furthermore, we postulated that the thinner LFW couldn't provide sufficient support for the DHS and further stress would concentrated on the medial wall. Then, coxa varus could occur. In this study, we found that the stress concentration area was located at the medial-inferior part of the proximal femur and the intertrochanteric fracture with a thicker LFW showed the smaller stress concentration compared with thinner LFW. The $10 \mathrm{~mm}$ model had the largest peek von Mises stress and the $20.5 \mathrm{~mm}$ model had the slightly smaller peek von Mises stress compared with $30 \mathrm{~mm}$ model. Therefore, we thought that the thinner of the LFW thickness, the higher risk of varus could occur in the intertrochanteric fractures after DHS fixation.

A decrease in the peak von Mises stress of the implant could decrease the risk of implant failure after daily loading. In this study, the stress distribution in the DHS was also evaluated. The stress in the DHS was concentrated near the junction of the barrel and side plate of each model. The maximum stress on the DHS increased by $44.25 \%$ and $47.52 \%$ when comparing $20.5 \mathrm{~mm}$ model and $10 \mathrm{~mm}$ model with $30 \mathrm{~mm}$ model, respectively. Therefore, the DHS of the $20.5 \mathrm{~mm}$ model and $10 \mathrm{~mm}$ model had higher risk of fatigue failure than the $30 \mathrm{~mm}$ model. Furthermore, the yield strength of Ti-6Al-7NB alloy was $921 \mathrm{MPa}$ [24], while the maximus stress in the DHS of the $10 \mathrm{~mm}$ model, $20.5 \mathrm{~mm}$ model and $30 \mathrm{~mm}$ model was $368.18 \mathrm{MPa}$, 360.03MPa and 249.58MPa, respectively. The maximum von Mises stress of the DHS was lower than the yield stress among all models. This mechanical property might reduce the breakage risk of the implant.

The total displacement of the fractured femur stabilized by a DHS device was used to evaluate the stability. In present study, the total displacement of the $10 \mathrm{~mm}$ model and $20.5 \mathrm{~mm}$ model increased by $20.59 \%$ and $16.19 \%$ when compared with $30 \mathrm{~mm}$ model, respectively. This indicated that a thicker LFW could increase the stability of the intertrochanteric fracture and the intertrochanteric fracture with a thinner LFW tended to increase the risk of failure after DHS fixation. Therefore, the intramedullary nail or an addition of trochanteric stabilization plate (TSP) was recommended with the intertrochanteric fractures with a thinner LFW.

To the best of the authors' knowledge, this was the first study to investigate the biomechanical performance of intertrochanteric fractures with different LFW thickness treated with DHS device. Nevertheless, there were several limitations in present study. First, the femur and implants were anisotropic materials. However, in this study, in order to reduce complexity of analysis, they were simplified into homogenous, isotropic and elastic materials. Second, only static analysis was conducted in this model, while dynamic analysis was ignored. More motion forms would be considered in the future research. Finally, this study did not conduct experimental validation, which definitely was a common limitation of similar simulation research. However, the purpose of this study was to compare relative values, and intertrochanteric fractures were difficult to achieve in vivo. Therefore, the lack of experimental validation was acceptable. This study analyzed the biomechanical performance of intertrochanteric fractures with different LFW thickness treated with DHS device only, and further research was needed to analyze more available fixation options (e.g., intramedullary nail). 


\section{Conclusions}

This work provided a biomechanical evaluation of DHS fixation for intertrochanteric fractures with different LFW thickness. This finite element modeling results revealed that the von Mises stress in the proximal femur, area around blade entry point and DHS were higher in the $10 \mathrm{~mm}$ model and $20.5 \mathrm{~mm}$ model when compared with the $30 \mathrm{~mm}$ model. Furthermore, the $30 \mathrm{~mm}$ model tended to have a smaller displacement when compared with the other two models. Therefore, the intertrochanteric fracture with a thinner LFW tended to have a higher risk of LFW fracture, implant failure and instability stabilized by a DHS device. Thus, the intertrochanteric fractures with a thinner LFW should not be treated by DHS alone and the intramedullary nail or an addition of trochanteric stabilization plate(TSP) was recommended. These findings from our study might provide a reference for the perioperative selection of internal fixations.

\section{Abbreviations}

DHS: dynamic hip screw; LFW: lateral femoral wall; FEA: finite element analysis; DICOM: Digital Imaging and Communications in Medicine; TAD: tip-apex distance; TSP: trochanteric stabilization plate

\section{Declarations}

\section{Ethics approval and consent to participate}

All procedures performed in studies involving human participants were in accordance with the ethical standards of the Ethical Committee of Peking University Third Hospital and with the 1964 Helsinki Declaration and its later amendments or comparable ethical standards. Written informed consent was obtained from all individual participants included in the study.

\section{Consent for publication}

Not applicable

\section{Availability of data and materials}

All data generated and analysed during this study are included in this article.

\section{Competing interests}

The authors declare that they have no competing interests.

\section{Funding}

This study was supported by Science and Technology fund of Winter Olympics (grant no. 2018YFF0301100); and Peking University Third Hospital (grant no. Y62419-06) 


\section{Authors' contributions}

Fang Zhou reviewed this study. Jixing Fan designed and participated in the whole process of the study and drafted the manuscript. Xiangyu Xu helped in the design and conduction of the study. All authors read and approved the final manuscript.

\section{Acknowledgements}

Not applicable

\section{References}

1. Adeyemi A and Delhougne G. Incidence and Economic Burden of Intertrochanteric Fracture: $A$ Medicare Claims Database Analysis. JB JS Open Access 2019; 4(1): e45.

2. Johnell $O$ and Kanis JA. An estimate of the worldwide prevalence, mortality and disability associated with hip fracture. Osteoporos Int 2004; 15(11): 897-902.

3. Burge R, Dawson-Hughes B and Solomon DH, et al. Incidence and economic burden of osteoporosisrelated fractures in the United States, 2005-2025. J Bone Miner Res 2007; 22(3): 465-475.

4. Gotfried Y. The lateral trochanteric wall: a key element in the reconstruction of unstable pertrochanteric hip fractures. Clin Orthop Relat Res 2004425): 82-86.

5. Palm H, Jacobsen S and Sonne-Holm S, et al. Integrity of the lateral femoral wall in intertrochanteric hip fractures: an important predictor of a reoperation. J Bone Joint Surg Am 2007; 89(3): 470-475.

6. Gupta RK, Sangwan K and Kamboj P, et al. Unstable trochanteric fractures: the role of lateral wall reconstruction. Int Orthop 2010; 34(1): 125-129.

7. Hsu CE, Shih $\mathrm{CM}$ and Wang CC, et al. Lateral femoral wall thickness. A reliable predictor of postoperative lateral wall fracture in intertrochanteric fractures. Bone Joint J 2013; 95-B(8): 1134-1138.

8. Pradeep AR, KiranKumar A and Dheenadhayalan J, et al. Intraoperative lateral wall fractures during Dynamic Hip Screw fixation for intertrochanteric fractures-Incidence, causative factors and clinical outcome. Injury 2018; 49(2): 334-338.

9. Henschel J, Eberle S and Augat P. Load distribution between cephalic screws in a dual lag screw trochanteric nail. J Orthop Surg Res 2016; 11(41.

10. Taylor ME, Tanner KE and Freeman MA, et al. Stress and strain distribution within the intact femur: compression or bending? Med Eng Phys 1996; 18(2): 122-131.

11. Kwak DK, Kim WH and Lee SJ, et al. Biomechanical Comparison of Three Different Intramedullary Nails for Fixation of Unstable Basicervical Intertrochanteric Fractures of the Proximal Femur: Experimental Studies. Biomed Res Int 2018; 2018(7618079.

12. Li J, Zhao $Z$ and Yin P, et al. Comparison of three different internal fixation implants in treatment of femoral neck fracture-a finite element analysis. J Orthop Surg Res 2019; 14(1): 76. 
13. Eberle $S$, Gerber $C$ and von Oldenburg $G$, et al. Type of hip fracture determines load share in intramedullary osteosynthesis. Clin Orthop Relat Res 2009; 467(8): 1972-1980.

14. Chen WP, Tai CL and Shih $\mathrm{CH}$, et al. Selection of fixation devices in proximal femur rotational osteotomy: clinical complications and finite element analysis. Clin Biomech (Bristol, Avon) 2004; 19(3): 255-262.

15. Lotz JC, Cheal EJ and Hayes WC. Stress distributions within the proximal femur during gait and falls: implications for osteoporotic fracture. Osteoporos Int 1995; 5(4): 252-261.

16. Lee PY, Lin KJ and Wei HW, et al. Biomechanical effect of different femoral neck blade position on the fixation of intertrochanteric fracture: a finite element analysis. Biomed Tech (Berl) 2016; 61(3): 331-336.

17. Kumar P, Rajnish RK and Sharma S, et al. Proximal femoral nailing is superior to hemiarthroplasty in AO/OTA $A 2$ and $A 3$ intertrochanteric femur fractures in the elderly: a systematic literature review and meta-analysis. Int Orthop 2020; 44(4): 623-633.

18. Chen W, Lv H and Liu S, et al. National incidence of traumatic fractures in China: a retrospective survey of 512187 individuals. Lancet Glob Health 2017; 5(8): e807-e817.

19. Socci AR, Casemyr NE and Leslie MP, et al. Implant options for the treatment of intertrochanteric fractures of the hip: rationale, evidence, and recommendations. Bone Joint J 2017; 99-B(1): 128-133.

20. Wu K, Xu Y and Zhang $L$, et al. Which implant is better for beginners to learn to treat geriatric intertrochanteric femur fractures: A randomised controlled trial of surgeons, metalwork, and patients. J Orthop Translat 2020; 21(18-23.

21. Ye KF, Xing $Y$ and Sun $C$, et al. Loss of the posteromedial support: a risk factor for implant failure after fixation of AO 31-A2 intertrochanteric fractures. Chin Med J (Engl) 2020; 133(1): 41-48.

22. Knobe M, Gradl $G$ and Ladenburger A, et al. Unstable intertrochanteric femur fractures: is there a consensus on definition and treatment in Germany? Clin Orthop Relat Res 2013; 471(9): 2831-2840.

23. Nie B, Chen $X$ and $L i ~ J$, et al. The medial femoral wall can play a more important role in unstable intertrochanteric fractures compared with lateral femoral wall: a biomechanical study. J Orthop Surg Res 2017; 12(1): 197.

24. Long M and Rack HJ. Titanium alloys in total joint replacement-a materials science perspective. Biomaterials 1998; 19(18): 1621-1639.

\section{Figures}




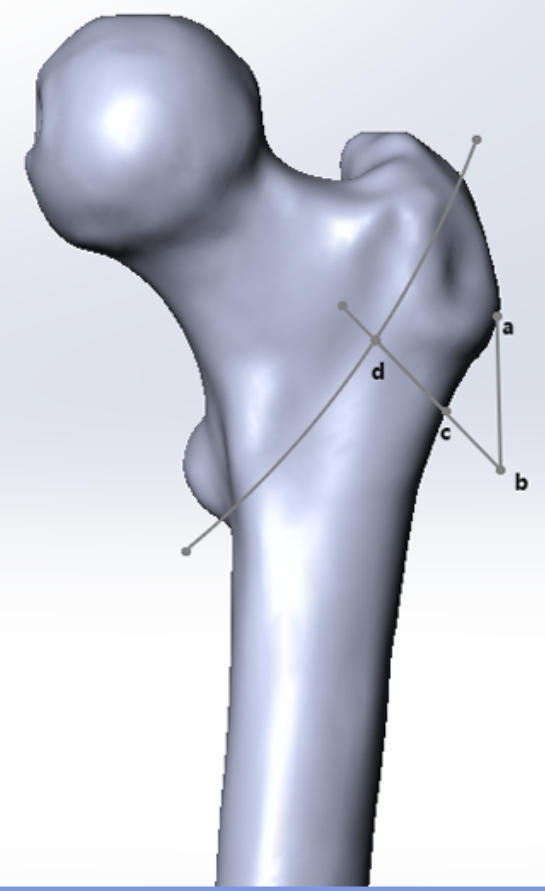

A

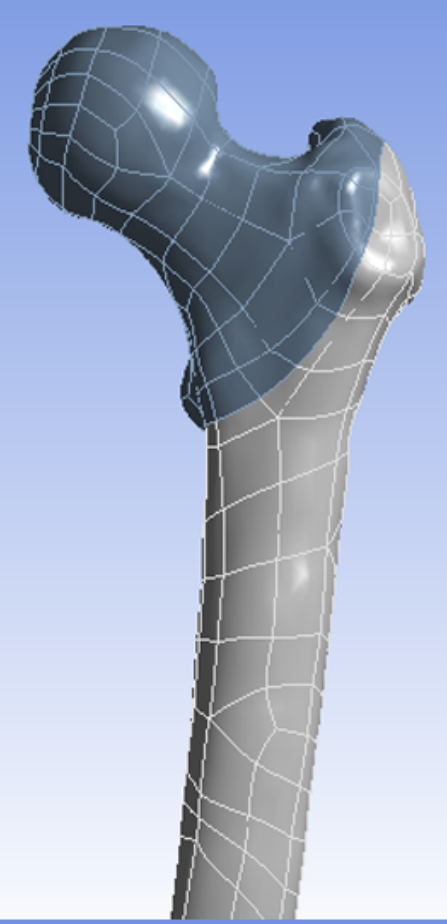

B
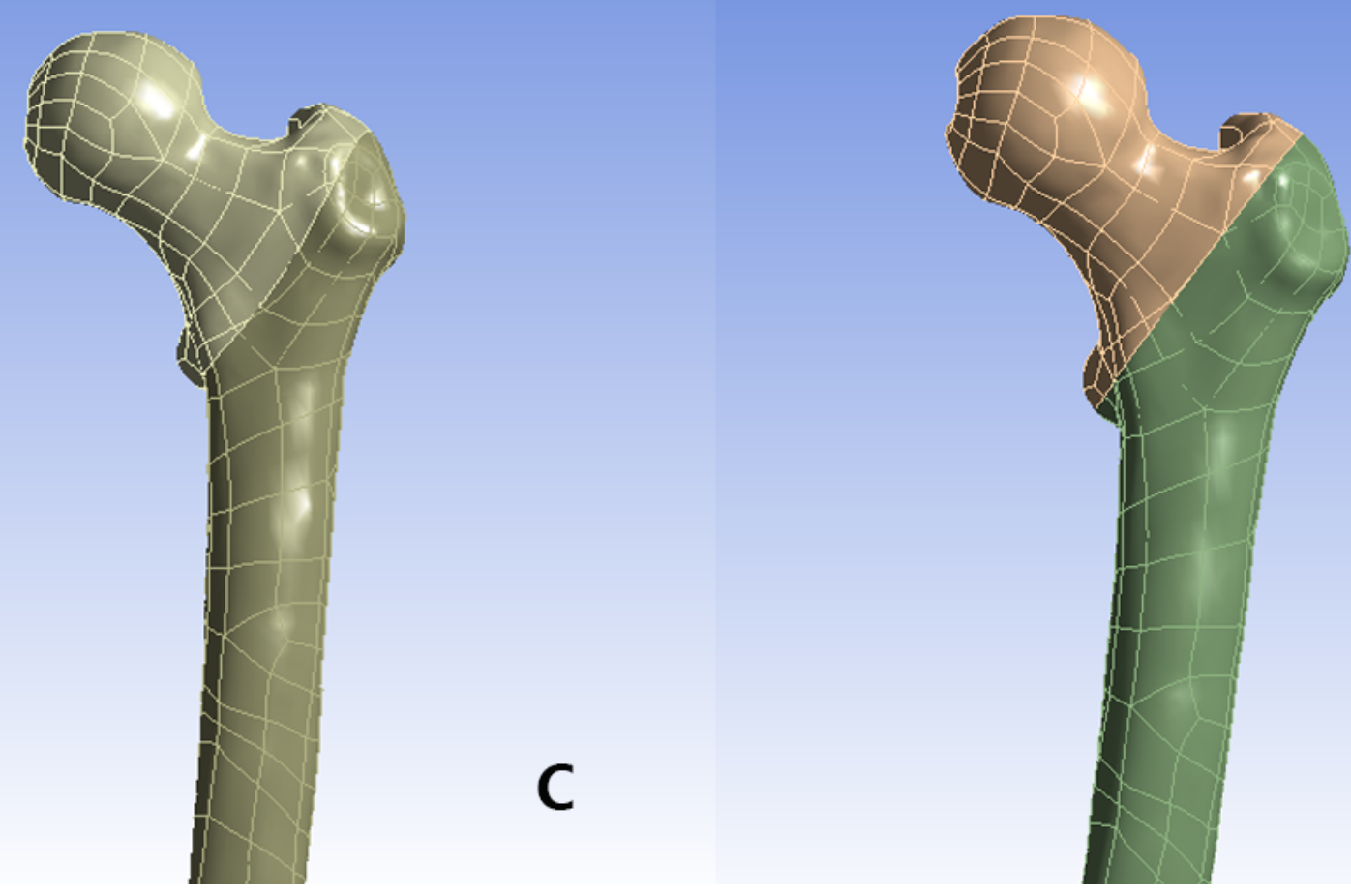

D

\section{Figure 1}

Three models of intertrochanteric fractures with different lateral femoral wall thickness were established. A. Diagram showing the lateral wall thickness (cd) defined as the distance in $\mathrm{mm}$ from a reference point $3 \mathrm{~cm}(\mathrm{ab})$ below innominate tubercle of greater trochanter, angled 135 upwards to the fracture line of the distal fragment on anteroposterior radiograph; B. The model of the intertrochanteric fracture with $10 \mathrm{~mm}$ LFW thickness; C. The model of the intertrochanteric fracture with $20.5 \mathrm{~mm}$ LFW thickness; D. The model of the intertrochanteric fracture with $30 \mathrm{~mm}$ LFW thickness. 

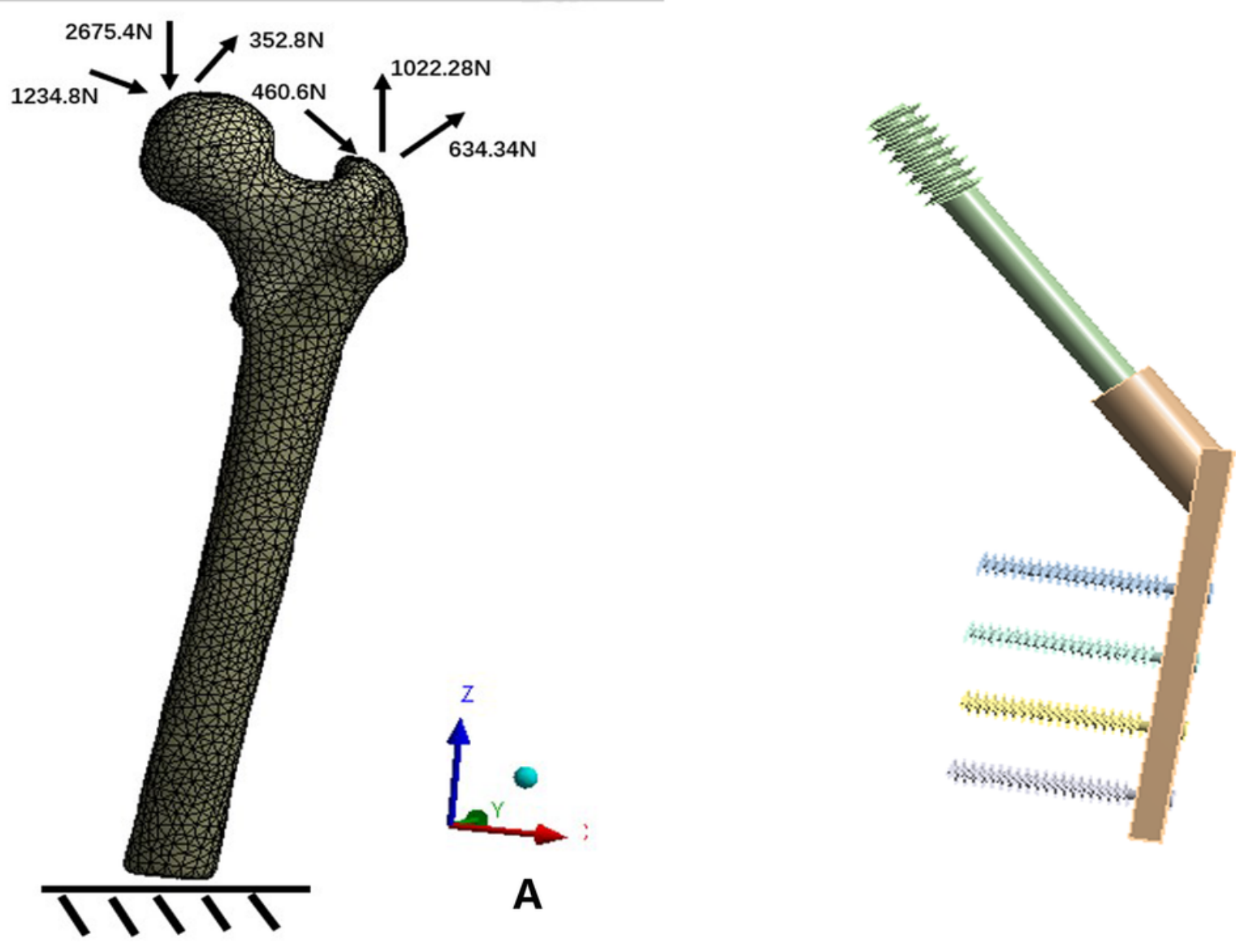

B

\section{Figure 2}

The meshed model of proximal femur and the loading acted on the model (A), model of dynamic hip screw (B)

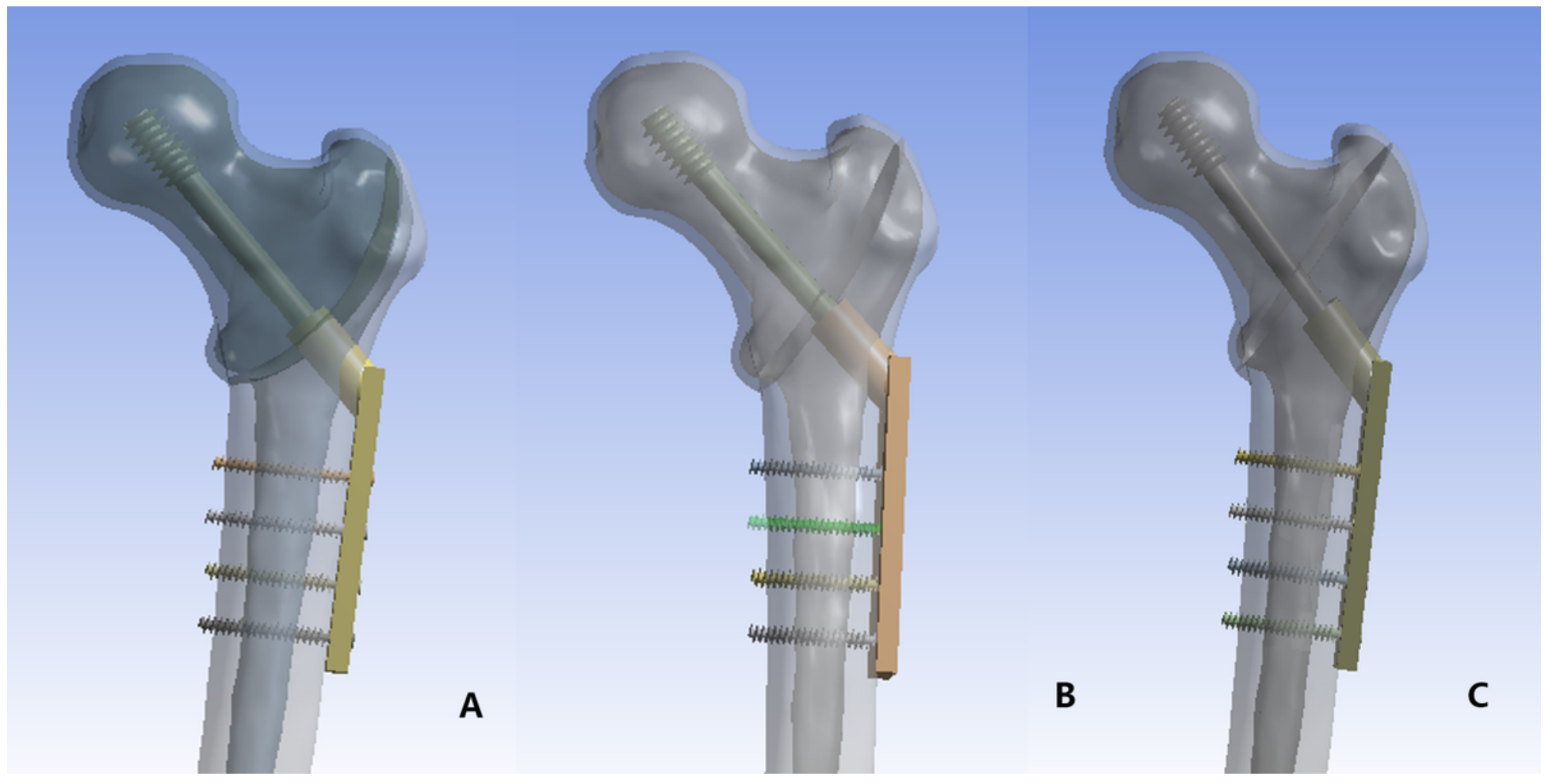


Figure 3

The model of intertrochanteric fracture with different lateral femoral wall thickness were established and implanted with the DHS. (A) $10 \mathrm{~mm}$ model; (B) $20.5 \mathrm{~mm}$ model; (C) $30 \mathrm{~mm}$ model

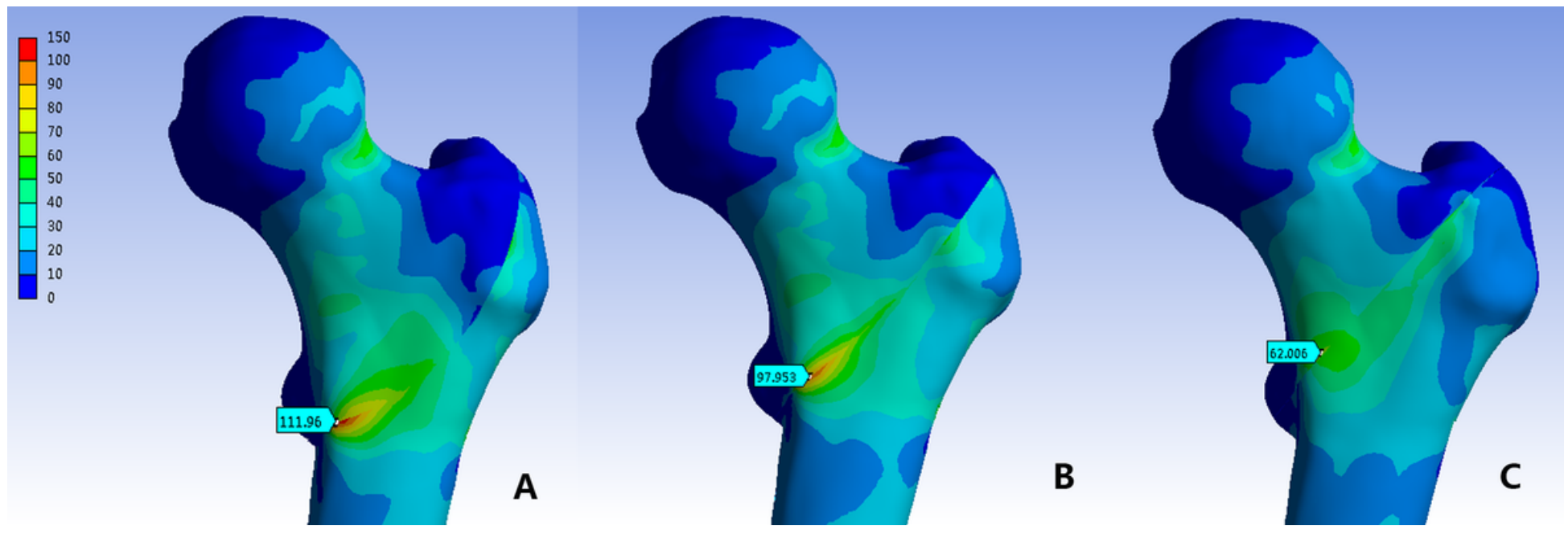

\section{Figure 4}

Von Mises stress distribution (MPa) on the proximal femur: (A) 10mm model; (B) 20.5mm model; (C) $30 \mathrm{~mm}$ model

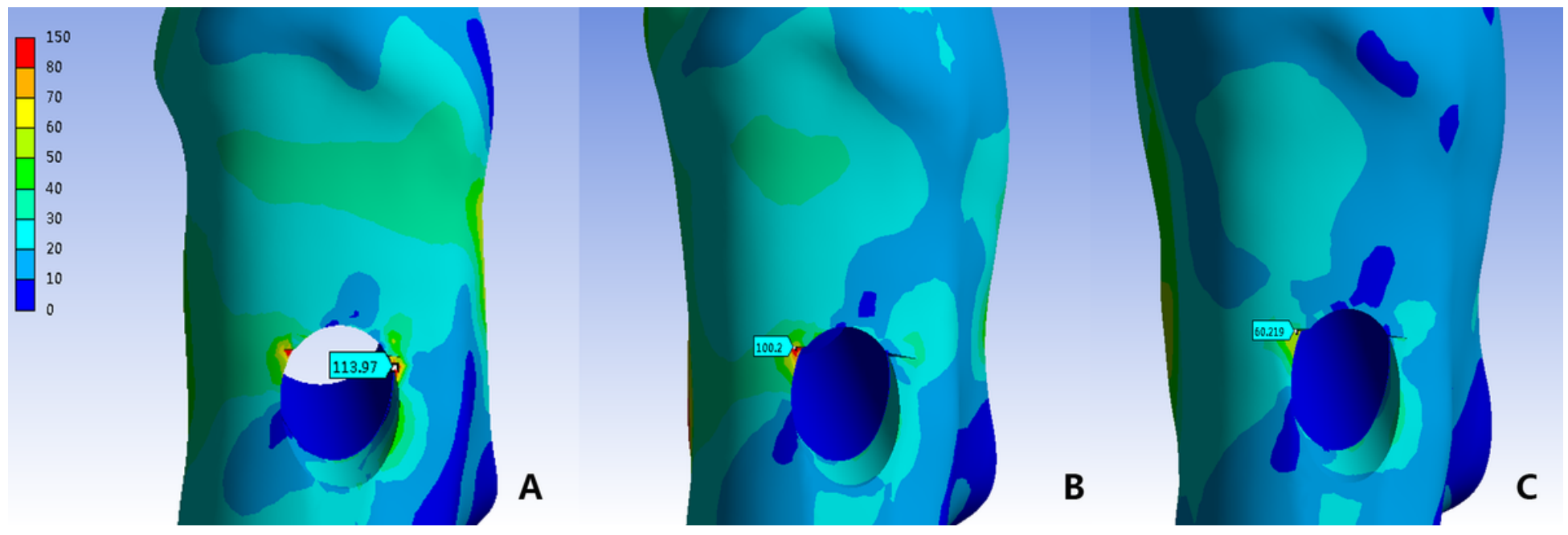

Figure 5

Von Mises stress distribution (MPa) on the lateral wall around blade entry point: (A) $10 \mathrm{~mm}$ model; (B) 20.5mm model; (C) $30 \mathrm{~mm}$ model 


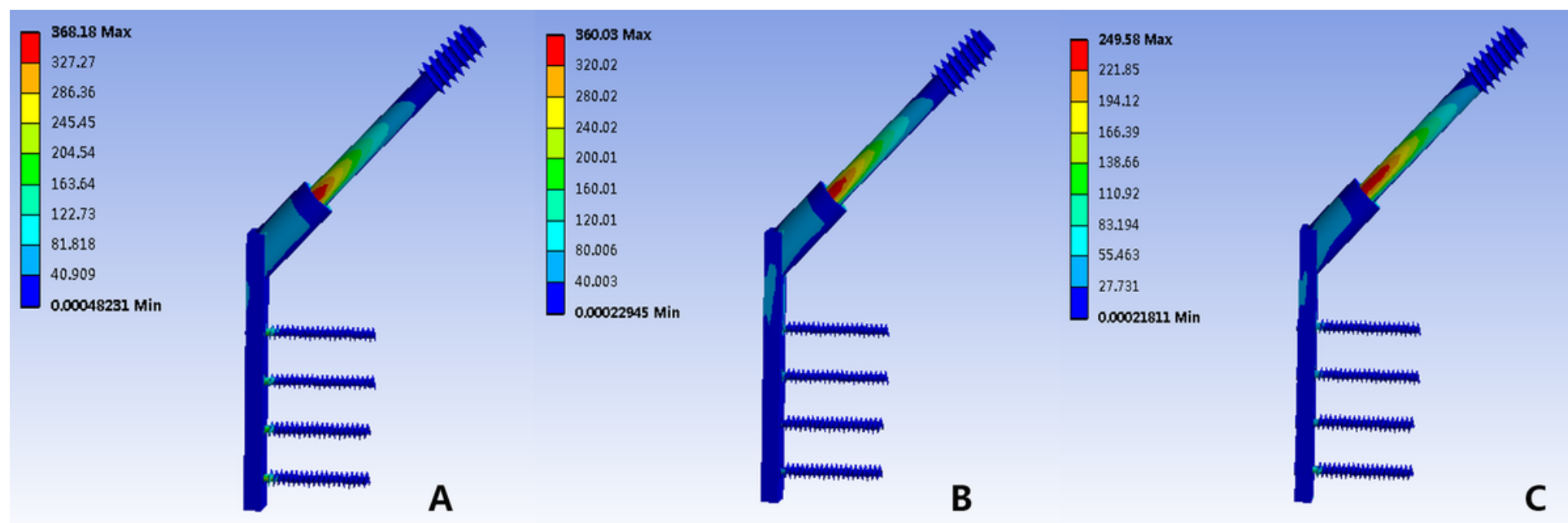

Figure 6

Von Mises stress distribution (MPa) on the DHS: (A) $10 \mathrm{~mm}$ model; (B) $20.5 \mathrm{~mm}$ model; (C) $30 \mathrm{~mm}$ model

$\square \begin{aligned} & 5.1944 \\ & 4.6172 \\ & 4.0401 \\ & -3.4629 \\ & 2.8858 \\ & 2.3086 \\ & -1.7315 \\ & 1.1543 \\ & 0.57716 \\ & 0\end{aligned}$
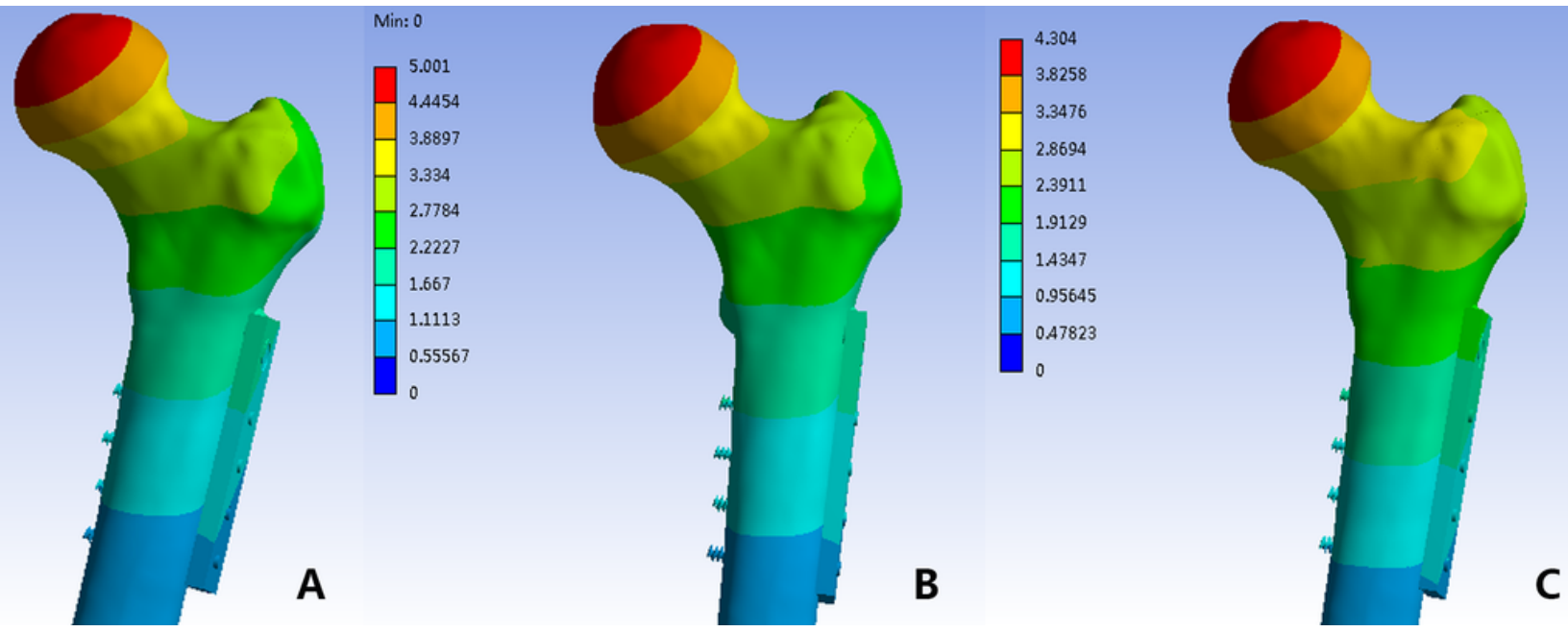

Figure 7

Displacement distribution ( $\mathrm{mm}$ ) in three models : (A) $10 \mathrm{~mm}$ model; (B) $20.5 \mathrm{~mm}$ model; (C) $30 \mathrm{~mm}$ model 